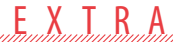 \\ Dementia \\ and Geriatric}

Cognitive Disorders
This is an Open Access article licensed under the terms of the Creative Commons AttributionNonCommercial-NoDerivs 3.0 License (www.karger.com/OA-license), applicable to the online version of the article only. Distribution for non-commercial purposes only.

\title{
Different Characteristics of Cognitive Impairment in Elderly Schizophrenia and Alzheimer's Disease in the Mild Cognitive Impairment Stage
}

\author{
Hiroaki Kazui ${ }^{a}$ Tetsuhiko Yoshidaa,b Masahiko Takaya ${ }^{a}$ Hiromichi Sugiyama ${ }^{a}$ \\ Daisuke Yamamoto ${ }^{a}$ Yumiko Kito ${ }^{a, c}$ Tamiki Wada $^{a}$ Keiko Nomura ${ }^{a}$ Yuka Yasuda ${ }^{a}$ \\ Hidenaga Yamamori $^{\mathrm{a}}$ Kazutaka Ohi $^{\mathrm{a}}$ Motoyuki Fukumoto $^{\mathrm{a}}$ Naomi like $^{\mathrm{a}}$

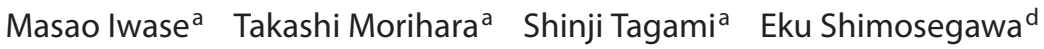

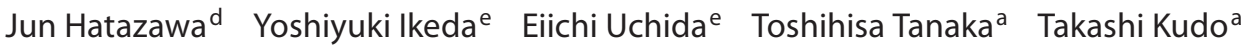 \\ Ryota Hashimoto ${ }^{a, f}$ Masatoshi Takeda ${ }^{a}$

\begin{abstract}
aPsychiatry, Department of Integrated Medicine, Division of Internal Medicine, Osaka University Graduate School of Medicine, ${ }^{\mathrm{b}}$ Department of Psychiatry, National Hospital Organization Osaka National Hospital, ' ${ }^{\circ}$ epartment of Psychiatry, Nissay Hospital, ${ }^{\mathrm{d}}$ Department of Nuclear Medicine and Tracer Kinetics, Osaka University Graduate School of Medicine, ${ }^{e}$ Department of Radiology, Uchida Clinic, and ${ }^{f}$ Molecular Research Center for Children's Mental Development, United Graduate School of Child Development, Osaka University, Kanazawa University and Hamamatsu University School of Medicine, Osaka, Japan
\end{abstract}

\section{Key Words}

Alzheimer's disease $\cdot$ Attention deficit • Delayed recall $\cdot$ Executive function $\cdot$ Recent memory $\cdot$ Three-dimensional stereotactic surface projections • Voxel-based specific region analysis • Working memory

\begin{abstract}
We compared indices of the revised version of the Wechsler Memory Scale (WMS-R) and scaled scores of the five subtests of the revised version of the Wechsler Adult Intelligence Scale (WAIS$R$ ) in 30 elderly schizophrenia (ES) patients and 25 Alzheimer's disease (AD) patients in the amnestic mild cognitive impairment (aMCl) stage (AD-aMCl). In the WMS-R, attention/concentration was rated lower and delayed recall was rated higher in ES than in $A D-a M C l$, although general memory was comparable in the two groups. In WAIS-R, digit symbol substitution, similarity, picture completion, and block design scores were significantly lower in ES than in AD$\mathrm{aMCl}$, but the information scores were comparable between the two groups. Delayed recall and


forgetfulness were less impaired, and attention, working memory and executive function were more impaired in $\mathrm{ES}$ than in $\mathrm{AD}-\mathrm{aMCl}$. These results should help clinicians to distinguish ES combined with $A D-a M C l$ from $E S$ alone.

Copyright $\odot 2011$ S. Karger AG, Basel

\section{Introduction}

Schizophrenia is a common psychiatric disease with onset usually occurring during adolescence or early adulthood. Recently, new atypical antipsychotic drugs for schizophrenia have been developed, and social systems to support schizophrenia patients have been established. As a result, schizophrenia patients are now living longer than they used to [1], and the number of elderly schizophrenia (ES) patients is increasing. The number of Alzheimer's disease $(\mathrm{AD})$ patients has also increased due to the rapid aging of society. Although the incidence of $\mathrm{AD}$ rises with age, $\mathrm{AD}$ also occurs in younger patients; the prevalence rate of $\mathrm{AD}$ in people aged $\leq 64$ years is 0.12 cases per 1,000 people (http://www.mhlw.go.jp/houdou/2009/03/h0319-2.html; Japanese Ministry of Health, Labor and Welfare). Therefore, there are many ES patients who also have $\mathrm{AD}$, and their number is supposed to be increasing. In clinical settings, there is a growing need to differentiate between age-related and ADrelated cognitive impairment in patients who have developed schizophrenia in adolescence or middle age.

Because some clinical characteristics of schizophrenia and $\mathrm{AD}$ are similar, differentiation between ES and AD can be difficult. Neuropsychiatric symptoms, such as apathy, poverty of speech, and delusional thinking, are common in both types of patients. Neuroimaging studies have shown volume loss in the hippocampus [2] and in the frontal lobe [3] in schizophrenia, and similar losses have been observed in AD [4]. Furthermore, patients with schizophrenia are impaired in various domains of cognition, such as memory, working memory, and executive function [5]. These symptoms are also observed in patients with AD.

Acetylcholine esterase inhibitors have been developed for the treatment of AD. Although administration of these agents does not result in a radical improvement of symptoms, their early administration can improve the prognosis of $\mathrm{AD}$ patients [6]. In addition, disease-modifying drugs for $\mathrm{AD}$ are now being developed. Thus, early diagnosis and early initiation of treatment are important in $\mathrm{AD}$ patients. One method to identify early $\mathrm{AD}$ with a high probability is the measurement of amnestic mild cognitive impairment (aMCI), which is a syndrome characterized by memory performance below the age norm, while intellectual functioning and activities of daily living are otherwise unimpaired [7]. A substantial proportion of patients with aMCI later develop clinically diagnosable AD [7]. In order to treat early-stage ES patients who have $\mathrm{AD}$ in the aMCI stage $(\mathrm{AD}-\mathrm{aMCI})$ for $\mathrm{AD}$, it is necessary to differentiate between ES combined with AD, and ES alone. As a first step toward this goal, in this study, we clarified the degree of cognitive impairment in patients with ES compared to patients with AD-aMCI.

\section{Methods}

\section{Subjects}

All patients in this study were recruited from the Department of Neuropsychiatry of the Osaka University Medical Hospital, which includes Schizophrenia and Neuropsychological Clinics. At both clinics, patients underwent standard neuropsychological examinations as well as routine laboratory tests and cranial magnetic resonance imaging (MRI). Single pho- 
Table 1. Comparison of characteristics of the ES and AD-aMCI groups with and without WAIS-R

\begin{tabular}{|c|c|c|c|c|c|c|}
\hline \multirow[t]{2}{*}{ Characteristics } & \multicolumn{3}{|l|}{ ES group } & \multicolumn{3}{|c|}{ AD-aMCI group } \\
\hline & with WAIS-R & without WAIS-R & $\mathrm{p}$ value & with WAIS-R & without WAIS-R & $\mathrm{p}$ value \\
\hline Sex, male/female & $5 / 9$ & $10 / 6$ & 0.14 & $7 / 6$ & $7 / 5$ & 0.57 \\
\hline Age, years & $56.6 \pm 5.5$ & $57.1 \pm 5.7$ & 0.79 & $72.6 \pm 6.0$ & $70.2 \pm 9.5$ & 0.44 \\
\hline Education, years & $13.1 \pm 2.6$ & $13.3 \pm 2.2$ & 0.79 & $13.7 \pm 3.3$ & $13.4 \pm 1.8$ & 0.8 \\
\hline MMSE total score & - & - & - & $26.1 \pm 1.9$ & $27.0 \pm 2.1$ & 0.27 \\
\hline WMS-R GM index & $81.3 \pm 15.5$ & $79.1 \pm 17.0$ & 0.75 & $80.5 \pm 13.1$ & $74.9 \pm 6.1$ & 0.19 \\
\hline WMS-R AC index & $84.8 \pm 10.3$ & $94.8 \pm 16.0$ & 0.09 & $99.8 \pm 11.1$ & $97.3 \pm 12.7$ & 0.59 \\
\hline WMS-R DR index & $75.9 \pm 15.9$ & $76.6 \pm 18.4$ & 0.92 & $61.5 \pm 9.7$ & $55.8 \pm 6.5$ & 0.1 \\
\hline
\end{tabular}

ton emission computed tomography (SPECT) was performed on patients with aMCI at the Neuropsychological Clinic. The clinical and investigative data were collected in a standardized manner and were entered into each registry. In this study, we selected patients with ES and patients with $\mathrm{AD}$-aMCI who met the inclusion criteria mentioned below for each group from the registry. In the Schizophrenia Clinic, we began using the revised version of the Wechsler Adult Intelligence Scale (WAIS-R) in March 2004 and then switched to the third version of the WAIS (WAIS-III) in October 2006. In the Neuropsychological Clinic, we began using five subtests of the WAIS-R in September 2002 and switched to five subtests of the WAIS-III in February 2009. In this study, we selected patients who were evaluated with the WAIS-R, because few patients with AD-aMCI were evaluated with the WAIS-III and then followed up until they reached the dementia stage. The revised version of the Wechsler Memory Scale (WMS-R) has been used in both clinics as a memory test because the third version of the WMS (WMS-III) is not standardized and cannot be used in Japan. In both clinics, the WMS-R was usually used before the WAIS-R. However, in some cases, there was no opportunity to use the WAIS-R.

\section{ES Group}

Thirty patients with schizophrenia (15 women and 15 men) were selected from the Schizophrenia Clinic registry. The mean age of the patients was $56.9 \pm 5.5$ years, and the mean years of education were $13.2 \pm 2.3$. All subjects in the ES group (1) met the criteria for schizophrenia based on the Structured Clinical Interview of the Diagnostic and Statistical Manual of Mental Disorders, 4th ed., Text Revision (DSM-IV-TR); (2) were aged $\geq 50$ years [8]; (3) showed first symptoms of schizophrenia before 65 years of age; (4) had been evaluated by either the WMS-R or the WAIS-R; (5) had no other neurological disease, and (6) had no evidence of focal brain lesions on MRI. Of the 30 patients, 14 were given the WAIS-R (group with WAIS-R) and the other 16 were not given the WAIS-R (group without WAIS-R). There were no significant differences in gender, age, education, or WMS-R indices between the ES groups with and without WAIS-R (table 1). Other demographic data on the ES group are summarized in table 2. Mean duration of hospitalization was short, although mean duration of disease was long. Many patients received atypical antipsychotic drugs at the time of neuropsychological assessment in this study. There were no significant differences between the groups with and without WAIS-R in any of the items except for the positive/negative symptom scores of the Positive and Negative Syndrome Scale (PANSS). Both PANSS scores were higher in the group without WAIS-R than in the group with WAIS-R. Four of the 30 patients with ES were not given the WMS-R. 
Table 2. Characteristics of the ES group

\begin{tabular}{|c|c|c|c|c|}
\hline Characteristics & $\begin{array}{l}\text { ES with WAIS-R } \\
\text { mean } \pm S D\end{array}$ & $\begin{array}{l}\text { ES without WAIS-R } \\
\text { mean } \pm S D\end{array}$ & $\begin{array}{l}\mathrm{p} \\
\text { value }\end{array}$ & $\begin{array}{l}\text { Total } \\
\text { mean } \pm \text { SD (range) }\end{array}$ \\
\hline Age of disease onset, years & $32.3 \pm 12.0$ & $30.1 \pm 12.3$ & 0.64 & $31.1 \pm 12.0(19.0-61.0)$ \\
\hline Duration of untreated psychosis, years & $3.6 \pm 6.5$ & $4.1 \pm 8.4$ & 0.87 & $3.9 \pm 7.5(0-26)$ \\
\hline Duration of disease, years & $23.8 \pm 11.7$ & $27.4 \pm 10.7$ & 0.41 & $25.8 \pm 11.1(1-45)$ \\
\hline Total duration of hospitalization, months & $14.0 \pm 12.2$ & $9.7 \pm 19.6$ & 0.56 & $11.4 \pm 16.8(0-72)$ \\
\hline Daily dose of antipsychotic drugs & & & & \\
\hline (chlorpromazine equivalent), mg & $554.7 \pm 283.6$ & $469.1 \pm 387.6$ & 0.5 & $509.0 \pm 340.0(0.0-1,300.0)$ \\
\hline Daily dose of atypical antipsychotic drugs & & & & \\
\hline $\begin{array}{l}\text { (chlorpromazine equivalent), mg } \\
\text { PANSS score }\end{array}$ & $485.7 \pm 306.6$ & $318.8 \pm 379.9$ & 0.2 & $396.7 \pm 352.0(0.0-1,300.0)$ \\
\hline Positive symptoms & $12.3 \pm 4.6$ & $16.3 \pm 4.4$ & 0.03 & $14.5 \pm 4.8(5-28)$ \\
\hline Negative symptoms & $12.3 \pm 3.2$ & $18.3 \pm 6.5$ & 0.01 & $15.5 \pm 6.0(7-30)$ \\
\hline $\begin{array}{l}\text { Overall severity in the Drug-Induced Extra- } \\
\text { Pyramidal Symptoms Scale }(n=21)\end{array}$ & $0.90 \pm 1.9$ & $0.86 \pm 0.7$ & 0.94 & $0.88 \pm 1.3(0-6)$ \\
\hline
\end{tabular}

\section{AD-aMCI Group}

Twenty-five AD-aMCI patients were selected from the Neuropsychological Clinic registry. The number of males exceeded the number of females (14 males and 11 females). The mean age of the patients was $71.4 \pm 7.8$ years, the mean years of education were $13.6 \pm 2.6$, and the mean MMSE score was $26.5 \pm 2.0$. All subjects in the AD-aMCI group met the criteria for aMCI, which included (1) a memory complaint documented by the patient or another source; (2) a score in the story A recall task in the logical memory II subtest of WMS$\mathrm{R}$ which is less than the age-corrected and education-corrected cutoff score; (3) a score of $\geq 24$ on the MMSE; (4) a total Clinical Dementia Rating (CDR) score of 0.5 and a memory CDR score $>0$; (5) normal basic and instrumental activities of daily living evaluated with Lawton's Physical Self-Maintenance Scale and Instrumental Activities of Daily Living Scale [9], and (6) no symptoms of dementia based on a clinical examination and an extensive interview with a knowledgeable informant. All subjects in this group also (7) had been evaluated by either the WMS-R or the short form of the Japanese version of the WAIS-R, (8) had no other neurological disease, and (9) had no evidence of focal brain lesions on MRI. To confirm that the aMCI patients had $\mathrm{AD}$ in the preclinical stage, at least one of the following three criteria had to be fulfilled: (1) atrophy in the entorhinal cortex on MRI, (2) hypoperfusion in the posterior cingulate cortex (PCC) and precuneus on SPECT, or (3) progression to AD during annual follow-ups. Progression to AD was defined as meeting the criteria of the National Institute of Neurological Disease and Stroke/Alzheimer's Disease and Related Disorders Association (NINCDS-ADRDA) for probable AD and a total CDR score of $\geq 1.0$.

Progression to AD from aMCI during the subsequent follow-ups (up to 8 years) was confirmed in 17 of the 25 patients. Nineteen of the $25 \mathrm{AD}$-aMCI patients received three-dimensional spoiled gradient echo MRI, which identified atrophy in the entorhinal cortex in 13 of the 19 patients. Twenty-three of the $25 \mathrm{AD}$-aMCI patients received $\mathrm{N}$-isopropyl-p-[ $\left.{ }^{123} \mathrm{I}\right]$-iodoamphetamine ( ${ }^{123}$ I-IMP)-SPECT, and hypoperfusion in either the PCC or precuneus was identified in 12 of the $23 \mathrm{AD}$-aMCI patients. One patient was recruited due to abnormality on the MRI and 7 patients were recruited due to abnormality on SPECT. Of the 25 patients, 13 were given the five subtests of the WAIS-R (group with WAIS-R) but the other 12 were not (group without WAIS-R). There were no significant differences in gender, age, education, 
Fig. 1. Z-score map overlaid on an MRI template of a representative patient with $\mathrm{AD}$-aMCI made with VSRAD. This patient was included in the study because of the presence of significant atrophy in the entorhinal cortices on MRI. Parts of the colored areas are in the areas circumscribed by purple lines, indicating significant atrophy in the entorhinal cortices. Purple lines indicate the bilateral entorhinal cortices. Colored areas on MRI are those with a Z-score $>2$ (significant atrophy). Color bar indicates $\mathrm{Z}$-score.

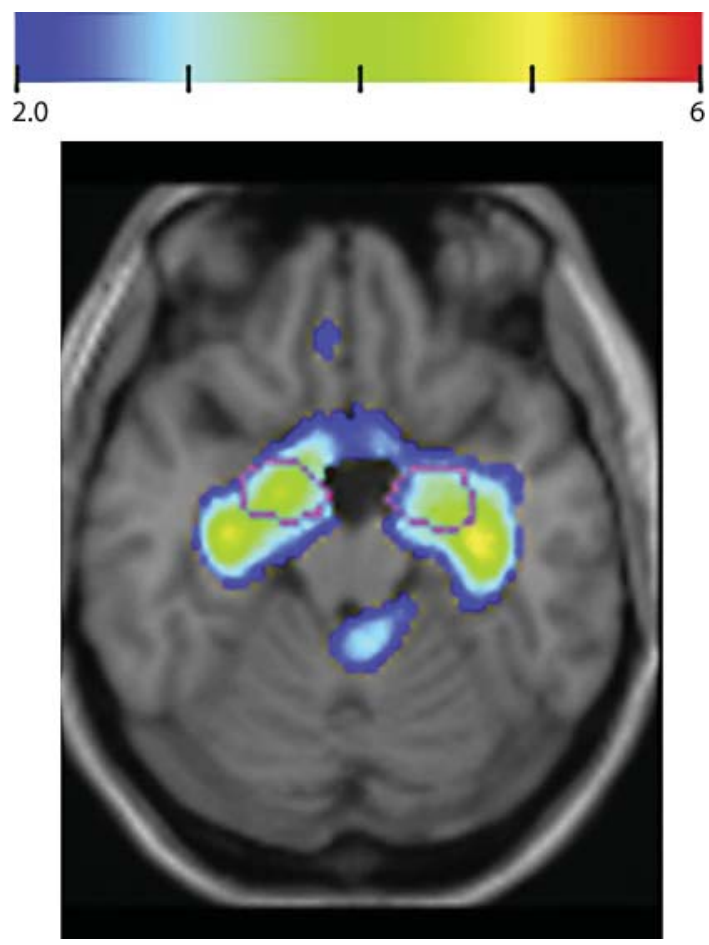

MMSE score or WMS-R indices between the two groups with and without WAIS-R (table 1). All AD-aMCI patients were administered the WMS-R.

\section{Comparison of Demographic Data in the ES and the AD-aMCI Groups}

There was no significant difference between the ES and the AD-aMCI groups in terms of $\operatorname{sex}\left(\mathrm{p}=0.48, \chi^{2}\right.$ test $)$ or education $(\mathrm{p}=0.71, \mathrm{t}$ test). However, the ES group was significantly younger than the AD-aMCI group $(\mathrm{p}<0.001$, t test).

\section{MRI and SPECT Criteria for the AD-aMCI Group}

MRI was performed on a 1.5-tesla system (Signa Excite HD 12x; General Electric Medical Systems, Milwaukee, Wisc., USA). A three-dimensional volumetric acquisition of a T1weighted gradient echo sequence produced a gapless series of thin sagittal sections that covered the whole calvarium. The operating parameters were as follows: field of view $=240 \mathrm{~mm}$, matrix $=256 \times 256,124 \times 1.40 \mathrm{~mm}$ contiguous sections, $\mathrm{TR}=12.55 \mathrm{~ms}, \mathrm{TE}=4.20 \mathrm{~ms}$, and flip angle $=15^{\circ}$. The three-dimensional T1-weighted MRI data of the patients were analyzed with the voxel-based specific region analysis for AD (VSRAD) [10] (fig. 1). VSRAD contained the MRI data of normal control subjects with a wide age range and could automatically compare the gray matter intensities of the MRI data on a voxel-by-voxel basis between an aMCI patient and age-comparable normal control subjects after a series of steps including segmentation, anatomical standardization and smoothing using Statistical Parametric Mapping 2002 (SPM2; Wellcome Department of Imaging Neuroscience, London, UK). The Z-score is calculated on a voxel-by-voxel basis as (Is - Ic)/SD where Is and Ic are the gray matter intensities of an aMCI patient and the mean of normal control subjects, respectively, and SD is the standard deviation of the gray matter intensities of the normal control subjects. The region of interest was set to the entorhinal cortex in the VSRAD software. Atrophy corresponding to a Z-score $>2.0$ in the entorhinal cortex was used as a criterion for AD in the VSRAD method. 


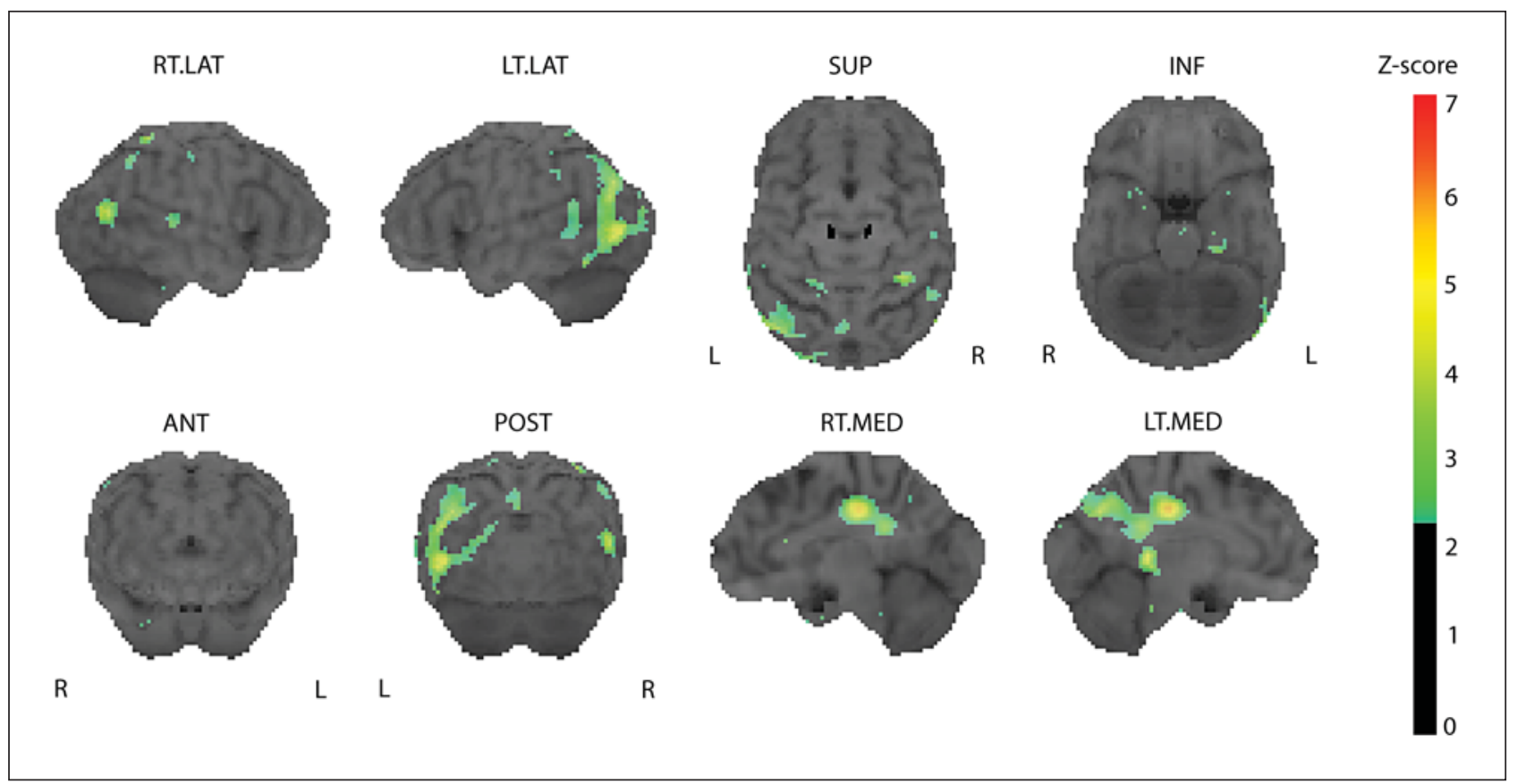

Fig. 2. Z-score map of a representative patient with AD-aMCI made with 3D-SSP. This patient was included in the study because of the presence of hypoperfusion in the PCC and precuneus on SPECT. Colored areas contain PCC and precuneus. Colored areas with significant rCBF reduction with a Z-score of $>2.32$ were overlaid on original surface images from eight views. Color bar indicates Z-score. RT.LAT = Right lateral; LT.LAT = left lateral; SUP = superior; INF = inferior; ANT = anterior; POST = posterior; RT.MED = right medial; LT.MED = left medial.

${ }^{123}$ I-IMP-SPECT was performed with a SPECT scanner (SPECT-2000H; Hitachi Medical Co., Tokyo, Japan) and a four-head rotating gamma camera. SPECT data were analyzed using three-dimensional stereotactic surface projection (3D-SSP) software [11] (fig. 2). 3D-SSP contained ${ }^{123}$ I-IMP-SPECT data of normal control subjects with a wide age range and could automatically compare the regional cerebral blood flow ( $\mathrm{rCBF}$ ) between an aMCI patient and age-comparable normal control subjects. The peak cortical values of the SPECT data were projected back and assigned to the original surface images from eight views on a pixel-bypixel basis. Z-score was calculated on a pixel-by-pixel basis as (Is - Ic)/SD where Is and Ic are the rCBFs of an aMCI patient and the mean of normal control subjects, respectively, and SD is the standard deviation of the $\mathrm{rCBF}$ of the normal control subjects. Areas with a Z-score $>2.32$ (the significance level of the Z-score) were overlaid on original surface images from eight views. With the computer program Stereotactic Extraction Estimation (SEE) we determined which gyri included the regions with a Z-score $>2.32$ [12]. In SEE, the percentage of areas with a Z-score $>2.32$ in each gyrus was calculated and the percentage was called the 'extent'. The presence of areas of hypoperfusion, in which both the Z-score was >2.32 and the extent was $>10 \%$ [13] in either the PCC or precuneus, was used as the inclusion criteria for $\mathrm{AD}$ in the aMCI stage.

\section{Assessment of Cognitive Functions}

The attention/concentration (AC) index in the WMS-R was used for measuring attention and working memory, the general memory (GM) index was used for recent memory, and the delayed recall (DR) index for delayed memory. For each index, the normal range is 
Table 3. Cognitive impairment in ES and $\mathrm{AD}$-aMC patients

\begin{tabular}{|c|c|c|c|}
\hline Test/subtest & ES group & AD-aMCI group & $\mathrm{p}$ value \\
\hline \multicolumn{4}{|l|}{$W M S-R$} \\
\hline GM index & $80.0 \pm 16.2$ & $77.8 \pm 10.5$ & 0.58 \\
\hline AC index & $91.0 \pm 14.7$ & $98.6 \pm 11.7$ & 0.046 \\
\hline DR index & $76.3 \pm 17.2$ & $58.8 \pm 8.6$ & $<0.001$ \\
\hline GM-DR & $3.6 \pm 10.7$ & $19.9 \pm 8.6$ & $<0.001$ \\
\hline \multicolumn{4}{|l|}{$W A I S-R$} \\
\hline Information & $10.1 \pm 3.7$ & $11.2 \pm 2.8$ & 0.37 \\
\hline Digit symbol substitution & $8.0 \pm 2.7$ & $11.6 \pm 2.3$ & $<0.001$ \\
\hline Similarity & $9.9 \pm 3.2$ & $12.5 \pm 2.2$ & 0.024 \\
\hline Picture completion & $8.5 \pm 4.0$ & $11.2 \pm 1.8$ & 0.037 \\
\hline Block design & $8.4 \pm 2.7$ & $11.5 \pm 1.9$ & 0.0018 \\
\hline
\end{tabular}

between 80 and 120 and the mean index of normal subjects is 100 . We also defined a new index equal to the GM index minus the DR index (GM-DR), which is a measure of the degree of forgetfulness.

For the WAIS-R, five test data were used in this study. Four of the five subtests were information, digit symbol substitution, similarities, and picture completion, which were selected according to the manual of the short form of the Japanese version of the WAIS-R [14]. Another was a block design to evaluate visuoconstructive function directly, as this dysfunction is a common symptom in AD patients. In each age-corrected score of the subtest, the normal range is between 7 and 13 and the mean score of normal subjects is 10 .

\section{Statistical Analyses}

Age-corrected scores of both the WMS-R and the five subtests of the WAIS-R were compared between the two groups using a t test. The significance level was set at $p<0.05$.

\section{Results}

Results of the WMS-R

In this study, the mean GM indices in the two groups were around the lower limit of the normal range, and the mean $\mathrm{AC}$ indices in $\mathrm{ES}$ and $\mathrm{AD}$-aMCI were normal (table 3). The mean DR index of ES was slightly below the normal range, but the mean DR index of ADaMCI appeared to be significantly lower. The GM indices of the two groups were comparable. The AC index was significantly lower and the DR index was significantly higher in ES than in AD-aMCI. The difference in the GM and DR scores (GM-DR), which is a measure of the degree of forgetfulness, was significantly lower in ES than in AD-aMCI.

\section{Results of the Five Subtests of the WAIS-R}

The mean scores of all the subtests of the WAIS-R in this study in both groups were within the normal range (table 3). The information scores of the two groups were comparable, but scores of the digit symbol substitution, similarity, picture completion, and block design subtests were significantly lower in ES than in AD-aMCI. 


\section{Discussion}

We could not confirm that all AD-aMCI patients in this study developed $\mathrm{AD}$ to the dementia stage. However, we were able to select aMCI patients that had AD-specific findings on MRI or SPECT in this study. Pathological abnormalities related to AD, neurofibrillary tangles and neuronal loss, were found to be present in the entorhinal cortex of AD in aMCI stage [15], leading to atrophy in the region on MRI [16]. Because the entorhinal cortex is functionally connected to the PCC [17], the reduction of rCBF in the PCC was probably caused by the abnormal pathology in the entorhinal cortex. In addition, atrophy in the entorhinal cortex on MRI [18] and reduction of $\mathrm{rCBF}$ in the PCC and precuneus on SPECT [19] predict progression from $\mathrm{MCI}$ to $\mathrm{AD}$. We used two reliable and user-independent statistical image-analyzing methods, VSRAD and 3D-SSP, to detect AD-specific abnormalities in the MR and SPECT images.

This is the first report to compare cognitive impairment between ES and AD-aMCI. The WMS-R GM indices of the two groups were comparable, indicating a similarity in the impairment of recent memory between the two groups. Some previous studies compared recent memory in ES and $\mathrm{AD}$ at the dementia stage. There is some disagreement on whether recent memory is better [20] or worse [21] in ES than in $\mathrm{AD}$ in the dementia stage. aMCI is a relatively homogeneous group with respect to memory impairment, because the definition of aMCI includes the degree of memory impairment. However, the severity of recent memory impairment could vary in patients with ES. The ES patients in this study were mild cases, because they could complete the WMS-R or WAIS-R, which are comprehensive tests, and the mean duration of their hospitalization was short. Thus, the recent memory tests in this study indicated that the recent memory scores of ES patients with mild cognitive impairment were comparable with those of $\mathrm{AD}$-aMCI patients, and, therefore, that recent memory was not useful for distinguishing between $\mathrm{ES}$ and $\mathrm{AD}-\mathrm{aMCI}$.

The fact that the WMS-R GM indices were comparable in the ES and AD-aMCI groups indicates that the two groups in this study had similar degrees of impairment of recent memory. This narrows down the difference between the two groups to differences in other cognitive impairments, such as forgetfulness, and impairments of DR, attention, working memory and executive function. The WMS-R GM-DR scores were lower and the DR scores were higher in $\mathrm{ES}$ than in $\mathrm{AD}$-aMCI, indicating that the degree of forgetfulness was less and DR was better in ES. On the other hand, the AC was lower in ES than in AD-aMCI, indicating that ES patients had more impaired attention and working memory than $\mathrm{AD}$-aMCI patients. $\mathrm{DR}$ was found to be better in ES patients than in $\mathrm{AD}$ patients in the dementia stage [21], and forgetfulness did not increase in ES patients but increased in AD patients in the dementia stage [20]. The present study confirmed that memory after a short while was retained in ES but not in $\mathrm{AD}$. In addition, we found that the retention in ES patients was better than in $\mathrm{AD}$ even at the aMCI stage, which should help to distinguish ES from AD in the very early stage.

The hippocampus, parahippocampus, and entorhinal cortex have traditionally been thought of as the principal structures responsible for the consolidation of short-term stores into long-term memory. Significant associations between hippocampal size and memory have not been observed in schizophrenia [22], although size reductions in the hippocampus have been reported in schizophrenia [2]. In addition, memory capabilities were similar to general intellectual abilities in ES [23]. Therefore, damage in the medial temporal lobe may not play an important role in memory impairment in schizophrenia. On the other hand, memory impairment in AD is inversely associated with hippocampal volume [24].

The ES group was more impaired on the digit symbol substitution, similarities, picture completion, and block design subtests of WAIS-R than the AD-aMCI group, and each subtest score in the ES group was below the mean of each score of the general population in this study. Although the block design subtest was used to evaluate visuoconstructive function in 
this study, attention and executive function are required to perform the block design subtest [25]. Thus, these findings confirmed that attention, working memory, and executive function are impaired in ES. Previous studies reported that ES patients were impaired in the WAIS-R digit symbol substitution, similarities, picture completion, and block design subtests [21], and in attention, working memory, and executive function [20]. These studies also reported that impairment in these functions were comparable in ES and AD patients in the dementia stage. The differences in cognitive impairment that we found in ES and AD-aMCI deviate from those found in previous studies. This discrepancy may be due to differences in the severity of cognitive impairments in the $\mathrm{AD}$-aMCI patients in this study compared to the $\mathrm{AD}$ patients in the dementia stage in previous studies.

Which region of the brain is responsible for the difference in attention, working memory, and executive function in the two groups? Impairments in cognitive function in patients with schizophrenia were found to be related to dysfunction of the prefrontal cortex (PFC) [26]. On the other hand, gray matter loss on MRI [27] and pathological abnormality [28] in the PFC were not observed in AD-aMCI, and gray matter loss on MRI was observed at the time of progression from aMCI to AD [27]. These results suggest that differences in impairment in attention, working memory, and executive function in the two groups probably reflect the difference in impairment in the PFC.

The WAIS-R information scores of the ES and AD-aMCI groups were comparable and within the normal range, being consistent with those of a previous study [29]. Semantic memory may be preserved in $\mathrm{ES}$ and $\mathrm{AD}$-aMCI patients because they have less impairment in the inferior and anterior temporal lobe regions, which crucially contribute to semantic cognition [30].

There were some limitations in this study. First, approximately half of the patients in each group were not given the WAIS-R. Second, the ES patients in this study were younger than the AD-aMCI patients, and cognitive function in schizophrenia patients undergoes a marked decline after 65 years of age [8]. Third, we did not control the effects of medication on the cognitive test scores in ES patients. Most ES subjects in this study had received atypical antipsychotic drugs, which might improve cognitive function [31]. These issues should be taken into consideration before the findings are generalized.

In this study, DR and forgetfulness were less impaired in ES than in AD-aMCI, while attention, working memory, and executive function were more impaired in ES than in ADaMCI. The results of this study should help clinicians to distinguish patients with ES from patients with $\mathrm{AD}-\mathrm{aMCI}$ and might also give us some clues for distinguishing ES combined with $\mathrm{AD}$-aMCI from ES alone. The next step is to clarify the difference in the characteristics of cognitive impairment in ES combined with $\mathrm{AD}$-aMCI compared to ES alone.

\section{Acknowledgments}

Funding for this study was provided by Research Grants for Research on Dementia (H21-Dementia-General-003 and H22-Dementia-General-003) and Grants-in-Aid (H19kokoro-002) from the Japanese Ministry of Health, Labor and Welfare, and by the Japanese Ministry of Education, Culture, Sports, Science and Technology (17191211, 18689030, 18023045, 20591402, and 21591514).

\section{Disclosure Statement}

The authors declare that they have no conflict of interest. 


\section{References}

1 Van Os J, Kapur S: Schizophrenia. Lancet 2009;374:635-645.

-2 Hirayasu Y, Shenton ME, Salisbury DF, Dickey CC, Fischer IA, Mazzoni P, Kisler T, Arakaki H, Kwon JS, Anderson JE, Yurgelun-Todd D, Tohen M, McCarley RW: Lower left temporal lobe MRI volumes in patients with first-episode schizophrenia compared with psychotic patients with first-episode affective disorder and normal subjects. Am J Psychiatry 1998;155:1384-1391.

-3 Goldman AL, Pezawas L, Mattay VS, Fischl B, Verchinski BA, Chen Q, Weinberger DR, Meyer-Lindenberg A: Widespread reductions of cortical thickness in schizophrenia and spectrum disorders and evidence of heritability. Arch Gen Psychiatry 2009;66:467-477.

-4 Laakso MP, Soininen H, Partanen K, Helkala EL, Hartikainen P, Vainio P, Hallikainen M, Hanninen T, Riekkinen PJ Sr: Volumes of hippocampus, amygdala and frontal lobes in the MRI-based diagnosis of early Alzheimer's disease: correlation with memory functions. J Neural Transm Park Dis Dement Sect 1995;9:73-86.

5 Dickinson D, Ramsey ME, Gold JM: Overlooking the obvious: a meta-analytic comparison of digit symbol coding tasks and other cognitive measures in schizophrenia. Arch Gen Psychiatry 2007;64: $532-542$.

-6 Winblad B, Wimo A, Engedal K, Soininen H, Verhey F, Waldemar G, Wetterholm AL, Haglund A, Zhang R, Schindler R: 3-year study of donepezil therapy in Alzheimer's disease: effects of early and continuous therapy. Dement Geriatr Cogn Disord 2006;21:353-363.

-7 Petersen RC, Smith GE, Waring SC, Ivnik RJ, Tangalos EG, Kokmen E: Mild cognitive impairment: clinical characterization and outcome. Arch Neurol 1999;56:303-308.

-8 Friedman JI, Harvey PD, Coleman T, Moriarty PJ, Bowie C, Parrella M, White L, Adler D, Davis KL: Six-year follow-up study of cognitive and functional status across the lifespan in schizophrenia: a comparison with Alzheimer's disease and normal aging. Am J Psychiatry 2001;158:1441-1448.

-9 Lawton MP, Brody EM: Assessment of older people: self-maintaining and instrumental activities of daily living. Gerontologist 1969;9:179-186.

-10 Hirata Y, Matsuda H, Nemoto K, Ohnishi T, Hirao K, Yamashita F, Asada T, Iwabuchi S, Samejima H: Voxel-based morphometry to discriminate early Alzheimer's disease from controls. Neurosci Lett 2005;382:269-274.

-11 Minoshima S, Berger KL, Lee KS, Mintun MA: An automated method for rotational correction and centering of three-dimensional functional brain images. J Nucl Med 1992;33:1579-1585.

-12 Mizumura S, Kumita S, Cho K, Ishihara M, Nakajo H, Toba M, Kumazaki T: Development of quantitative analysis method for stereotactic brain image: assessment of reduced accumulation in extent and severity using anatomical segmentation. Ann Nucl Med 2003;17:289-295.

-13 Kazui H, Ishii R, Yoshida T, Ikezawa K, Takaya M, Tokunaga H, Tanaka T, Takeda M: Neuroimaging studies in patients with Charles Bonnet syndrome. Psychogeriatrics 2009;9:77-84.

14 Misawa Y: Manual of the Short Form of the Japanese Version of the WAIS-R (in Japanese). Tokyo, Nihon Bunka Kagakusha, 1993.

-15 Gomez-Isla T, Price JL, McKeel DW Jr, Morris JC, Growdon JH, Hyman BT: Profound loss of layer II entorhinal cortex neurons occurs in very mild Alzheimer's disease. J Neurosci 1996;16:4491-4500.

-16 Killiany RJ, Gomez-Isla T, Moss M, Kikinis R, Sandor T, Jolesz F, Tanzi R, Jones K, Hyman BT, Albert MS: Use of structural magnetic resonance imaging to predict who will get Alzheimer's disease. Ann Neurol 2000;47:430-439.

-17 Mosconi L, Pupi A, De Cristofaro MT, Fayyaz M, Sorbi S, Herholz K: Functional interactions of the entorhinal cortex: an 18F-FDG PET study on normal aging and Alzheimer's disease. J Nucl Med 2004;45:382-392.

-18 Killiany RJ, Hyman BT, Gomez-Isla T, Moss MB, Kikinis R, Jolesz F, Tanzi R, Jones K, Albert MS: MRI measures of entorhinal cortex vs hippocampus in preclinical AD. Neurology 2002;58:11881196.

-19 Kogure D, Matsuda H, Ohnishi T, Asada T, Uno M, Kunihiro T, Nakano S, Takasaki M: Longitudinal evaluation of early Alzheimer's disease using brain perfusion SPECT. J Nucl Med 2000;41:1155-1162.

-20 Heaton R, Paulsen JS, McAdams LA, Kuck J, Zisook S, Braff D, Harris J, Jeste DV: Neuropsychological deficits in schizophrenics. Relationship to age, chronicity, and dementia. Arch Gen Psychiatry 1994;51:469-476. 
-21 Zakzanis KK, Andrikopoulos J, Young DA, Campbell Z, Sethian T: Neuropsychological differentiation of late-onset schizophrenia and dementia of the Alzheimer's type. Appl Neuropsychol 2003;10: 105-114.

-22 Torres IJ, Flashman LA, O’Leary DS, Swayze V 2nd, Andreasen NC: Lack of an association between delayed memory and hippocampal and temporal lobe size in patients with schizophrenia and healthy controls. Biol Psychiatry 1997;42:1087-1096.

-23 Hawkins KA: Memory deficits in patients with schizophrenia: preliminary data from the Wechsler Memory Scale-Third Edition support earlier findings. J Psychiatry Neurosci 1999;24:341-347.

-24 Mori E, Yoneda Y, Yamashita H, Hirono N, Ikeda M, Yamadori A: Medial temporal structures relate to memory impairment in Alzheimer's disease: an MRI volumetric study. J Neurol Neurosurg Psychiatry 1997;63:214-221.

-25 Chase TN, Fedio P, Foster NL, Brooks R, Di Chiro G, Mansi L: Wechsler Adult Intelligence Scale performance. Cortical localization by fluorodeoxyglucose F18-positron emission tomography. Arch Neurol 1984;41:1244-1247.

-26 Ikezawa K, Iwase M, Ishii R, Azechi M, Canuet L, Ohi K, Yasuda Y, Iike N, Kurimoto R, Takahashi H, Nakahachi T, Sekiyama R, Yoshida T, Kazui H, Hashimoto R, Takeda M: Impaired regional hemodynamic response in schizophrenia during multiple prefrontal activation tasks: a two-channel near-infrared spectroscopy study. Schizophr Res 2009;108:93-103.

-27 Whitwell JL, Przybelski SA, Weigand SD, Knopman DS, Boeve BF, Petersen RC, Jack CR Jr: 3D maps from multiple MRI illustrate changing atrophy patterns as subjects progress from mild cognitive impairment to Alzheimer's disease. Brain 2007;130:1777-1786.

-28 Petersen RC, Parisi JE, Dickson DW, Johnson KA, Knopman DS, Boeve BF, Jicha GA, Ivnik RJ, Smith GE, Tangalos EG, Braak H, Kokmen E: Neuropathologic features of amnestic mild cognitive impairment. Arch Neurol 2006;63:665-672.

-29 Kirkpatrick B, Messias E, Harvey PD, Fernandez-Egea E, Bowie CR: Is schizophrenia a syndrome of accelerated aging? Schizophr Bull 2008;34:1024-1032.

-30 Visser M, Embleton KV, Jefferies E, Parker GJ, Ralph MA: The inferior, anterior temporal lobes and semantic memory clarified: novel evidence from distortion-corrected FMRI. Neuropsychologia 2010;48:1689-1696.

- 31 Houthoofd SA, Morrens M, Sabbe BG: Cognitive and psychomotor effects of risperidone in schizophrenia and schizoaffective disorder. Clin Ther 2008;30:1565-1589. 